\title{
Thermodyamic and Kietic Study on the Corrosion of Aluminium in Hydrochloric Acid using Benzaldehyde as Corrosion Inhibitor
}

\author{
*Musa Husaini, Muhammad Bashir Ibrahim \\ ${ }^{a}$ Department of Pure and Industrial Chemistry ,Faculty of Physical Sciences, Bayero University, P.M.B. 3011 \\ BUK, Kano. Nigeria
}

Received: 07 September 2019; Accepted: 15 October 2019; Published: 08 November 2019

\begin{abstract}
The inhibition of the corrosion of aluminium by benzaldehyde in $1.4 \mathrm{M} \mathrm{HCl}$ was investigated using weight loss method and characterized by FT-IR analysis. The results showed that the corrosion rate of aluminium in $1.4 \mathrm{M} \mathrm{HCl}$ decreases with increase in concentration of the inhibitor. The inhibition efficiency increases progressively as the concentration of the inhibitor increases. Effects of temperature on the inhibition efficiency of the inhibitor showed that inhibition efficiency decreases with increase in temperature. The value of activation energy (Ea) was found to be $20.55 \mathrm{Kjmol}-1$ for aluminium corrosion in $1.4 \mathrm{M} \mathrm{HCl}$ which was increased to $34.49 \mathrm{Kjmol}-1$ in the presence of $0.1 \mathrm{M}$ inhibitor concentration. The calculated values for enthalpy of activation $(\Delta \mathrm{Ha})$ were all positive indicating the endothermic nature of the aluminium dissolution process. The obtained values of Gibbs free energy ( $\Delta$ Gads) was in the range of -17.94 to $-18.27 \mathrm{~kJ}$ mol-1. Kinetics of the reaction in the presence of the inhibitor revealed that it follows a first order reaction. The value of rate constant $(\mathrm{k})$ was reduced from uninhibited acid to the inhibited acid solution, while the half-life values in the presence of the inhibitor were higher compared to the value in uninhibited acid solution suggesting that inhibition efficiency increases with increase in the concentration of the inhibitor.
\end{abstract}

Index Terms: Aluminum, benzaldehyde, FT-IR analysis, kinetic parameters, thermodynamic parameters and weight loss.

(C) 2019 Published by MECS Publisher. Selection and/or peer review under responsibility of the Research Association of Mode rn Education and Computer Science

\footnotetext{
* Corresponding author.

E-mail address: musahusaini36@gmail.com
} 


\section{Introduction}

Aluminium is the most widely used non-ferrous metal in all the fields of domestic and industrial applications like construction, transportation, packaging, a wide range of household items, electrical transmission and outer shell of consumer electronics etc. Aluminium is remarkable for its low density and ability to resist corrosion due to the passivation phenomenon [1]. The protective coating of aluminium destroyed when exposed to acid or alkaline environment and corrosion of aluminium occurs, yielding $\mathrm{Al}+3$ ion in acid solution and $\mathrm{AlO} 2$ in the alkaline solution [2]. Acid pickling is usually used for aluminium surface treatment. Aluminium can react with hydrochloric acid to produce hydrogen gas. The chloride ions of the $\mathrm{HCl}$ cause a substantial loss of the metal by corrosion and Cl- ions of the acid create extensive localized attack over the surface of aluminium. Hence to avoid such kind of attack on the base metal during pickling and cleaning aluminium surfaces with acidic solutions, the use of inhibitors become necessary. The use of inhibitors is among the most practical methods for protection against corrosion in acidic media. Various organic compounds are reported as good corrosion inhibitors for aluminium in hydrochloric acid media [3, 4]. A great number of research have being devoted to the subject of corrosion inhibitors in both the laboratory and fields. Corrosion inhibition is of great practical importance, as it's extensively employed in shortening wastage of engineering materials and reducing corrosion control costs. Inhibitor applications is quite varied usually playing an important role to minimize localized corrosions and unexpected sudden failures in water treatment facility, oil extraction and processing industries, ferrous metal cleaners, heavy industrial manufacturing, water treatment chemicals, water-containing hydraulic fluids, automatic transmission fluids, automotive component manufacture, cutting fluids, engine coolants etc. Most of the well-known acid inhibitors are organic compounds containing nitrogen, oxygen, phosphorus, sulfur and $\pi$ bonds, as well as aromatic rings in their structure which are the major adsorption centers [5]. Generally, a strong interaction causes higher inhibition efficiency, the inhibition effect increases in the sequence $\mathrm{O}<\mathrm{N}<\mathrm{S}[6,7]$. Compounds with $\pi$-bonds also exhibit good inhibitive properties due to interaction of $\pi$ orbital with metal surface. The objectives of the present research is to study the kinetic parameters, thermodynamic parameters, adsorption isotherm and the inhibition effect of benzaldehyde as inhibitor on the corrosion of aluminium in hydrochloric acid at different inhibitor concentration and temperature. The weight loss method was used in this study as its quantitative and possibly most accurate method in monitoring and measuring corrosion of metallic structures.

\subsection{Significance of the Research}

The investigation of the corrosion inhibition of benzaldehyde on aluminium provided a useful information on surface coverage, inhibition efficiency, thermodynamic and kinetic data, and the type of adsorption of benzaldehyde inhibitor on the surface of aluminium. The use of benzaldehyde inhibitor will reduce the rates of usage of inorganic inhibitors which are toxic to environment. This submission is in advancement of contribution toward the sustained world-wide in looking for friendly, non-toxic and commercially available corrosion inhibitors.

\subsection{Literature Review}

Several report have been made to the organic compounds that contain nitrogen, sulfur and nitrogen as polar groups and conjugated double bonds in their structures to be good corrosion inhibitors for many metals and alloys in corrosive environment [8]. The inhibiting effect of these organic compounds is usually attributed 
to their interactions with the metallic surfaces through their adsorption. Polar functional groups serves as the reaction center that stabilitizes the adsorption process. Nevertheless the adsorption of an inhibitor on a metal surface depends on certain factors which include; the inhibitor's chemical structure, nature and surface charge of the metal, the type of the electrolyte solution and the adsorption mode [9].

The Inhibition of Aluminum corrosion in $3.5 \% \mathrm{NaCl}$ solution using diisopropyl thiourea (DISOTU) has been reported by Karthikeyan et al [10] using weight loss, electrochemical polarization technique, impedance method and quantum mechanical measurement. It was found that the aluminium corrosion in the sea water medium was minimize effectively by the compound. Adsorption of the compound on metal was noticed to follow Langmuir adsorption isotherm. The inhibition efficiency (IE) increases with increase in inhibitor concentration. The adsorption of protective layer of inhibitor on aluminium surface was confirmed by using Quantum mechanical studies.

The effect of formazan of benzaldehyde (FB) on the corrosion of mild steel in acidic media $(1.0 \mathrm{M} \mathrm{HCl}$ and $2 \mathrm{M} \mathrm{HCl}$ ) has been investigated by Anand [11] using weight loss measurements, electrochemical studies and surface analysis. These studies have also shown that formazan of benzaldehyde is a good inhibitor for mild steel in $1.0 \mathrm{M} \mathrm{HCl}$ and $2 \mathrm{M} \mathrm{HCl}$ acid solutions at room temperature in $2 \mathrm{~h}$. In $1.0 \mathrm{M} \mathrm{HCl}$ the inhibition efficiency was high when compared to $2 \mathrm{M} \mathrm{HCl}$ acid solutions. The surface analysis also confirms the corrosion inhibition of the mild steel by the inhibitor (FB).

Aluminium and its alloys are vitally preferred materials of construction in many chemical and engineering fields due to their light weight corrosion resistant properties. Nitrogen-containing organic compounds, like amines and diamine derivatives offer good protection of metallic materials on the corrosion for many metals in acidic solutions. Arvnabh et al [12] analyzed the corrosion inhibition of aluminium alloys of grade 1060, 1100 and 3003 in trichloroacetic by using conductivity and potentiostatic polarization in different inhibitors of diamine such as ethyl amino ethylamine, di-methyl amino ethylamine, 1:3 di-amino propane, tetra methyl ethylene diamine. Diamines are act as mixed inhibitors as revealed by polarization curves in the case of alloy of 3003 grade.

In the recent investigation effect of Triethylenetetramine [TETA] and 2-(2-aminoethylamino) ethanol [AEAE] as corrosion inhibitors for $\mathrm{N} 80$ steel in $15 \% \mathrm{HCl}$ solution was studied by Yadav et al [13] using polarization, AC impedance (EIS) and weight loss measurements. Both inhibitors were found to be effective inhibitors. Inhibition efficiency was found to increase significantly with increasing inhibitor concentration. Mixed-type inhibitors were revealed from polarization studies. Charge transfer resistance increases and double layer capacitance decreases in presence of inhibitors as revealed by AC impedance studies. Inhibitors adsorption on the surface of N80 steel follows Langmuir adsorption isotherm.

\section{Material and Methods}

\subsection{Specimen Preparation}

Rectangular specimen of aluminium (99.5 \%) obtained from Metal Focus Fabrication Technology Incubation Centre Kano State, Nigeria was used in this study [14]. The specimen of size $3 \times 2 \times 0.1 \mathrm{~cm}$ with a small hole near the upper edge was used for the determination of corrosion rate. The specimen was polished using different grade of emery paper, degreased in absolute ethanol, dried in acetone and stored in a moisture-free desiccators prior to use.

\subsection{Preparation of Solutions}


Double distilled water was used to prepare a stock solution of analytical grade hydrochloric acid $(36.5 \%$, $1.18 \mathrm{~g} / \mathrm{cm} 3)$. The required concentration of the acid solution was prepared by appropriate dilutions. The used inhibitor was benzaldehyde $(95 \% 1.04 \mathrm{~g} / \mathrm{cm} 3)$, and concentrations of the inhibitor used for the study was 0.02 , 0.04, 0.06, 0.08 and $0.1 \mathrm{M}$. Each of these concentrations was diluted in the prepared desired concentrations of acids for use as test solutions in weight loss.

\subsection{Weight loss Measurement}

The prepared weighted specimens were immersed in $100 \mathrm{ml}$ beaker containing the acid solution in the absence and presence of various concentrations of the inhibitor $(0.02,0.04,0.06,0.08 \& 0.1 \mathrm{M})$ at $308 \mathrm{~K}$ and $3 \mathrm{hrs}$ immersion time, after which they were retrieved, washed, dried, re-weighted and recorded. The experiment were performed in replicate. The effect of temperature was studied at a temperature range of 308 , 313 and $318 \mathrm{~K}$. The weight loss of aluminium was calculated in grams as the difference between the initial weight and the weight after the removal of the corrosion product. The weight loss $(\Delta \mathrm{w})$ corrosion rate (C.R), inhibition efficiency (I.E) and degree of surface coverage $(\Theta)$ were calculated using the Equations 1, 2, 3 and 4 respectively.

$$
\begin{aligned}
& \Delta \mathrm{W}=\mathrm{W}_{1}-\mathrm{w}_{\mathrm{f}} \\
& \text { C.R }=\frac{W_{1}-W_{\mathrm{f}}}{\mathrm{At}} \\
& \Theta=\frac{W_{0}-W_{1}}{W_{0}} \\
& \text { I.E }=\frac{W_{0}-W_{1}}{W_{0}} \times 100
\end{aligned}
$$

Where wi and wf are the initial and final weight of Aluminium samples, w1 and w0 are the weight loss values in presence and absence of inhibitor, respectively. $\mathrm{A}$ is the total area of the aluminium specimen and $\mathrm{t}$ is the immersion time.

\subsection{Fourier Transform Infrared Spectroscopic Analysis}

FT-IR analysis was carried out for the fresh inhibitor and that of the corrosion product obtained from the reaction of aluminium immersed in $1.4 \mathrm{M} \mathrm{HCl}$ solution for $3 \mathrm{hrs}$ immersion time in the presence of $0.1 \mathrm{M}$ benzaldehyde at $308 \mathrm{~K}$ using Agilent Technology, FTIR (Cary 630) Fourier Transform Infrared Spectrophotometer. $650-4000 \mathrm{~cm}^{-1}$ wave number was used to scan the sample during the analysis.

\section{Results and Discussion}

The data of all figures presented in this section are obtained from the result presented in Table 1.

\subsection{Effect of inhibitor on corrosion rate and inhibition efficiency}


Figure 1 shows the plot of corrosion rate against inhibitor concentration for aluminium corrosion in $1.4 \mathrm{M}$ $\mathrm{HCl}$ at different temperatures. The figure reveals that the rate of corrosion of aluminium in $1.4 \mathrm{M}$ hydrochloric acid decreases with increase in inhibitor concentration at all the temperatures studied. The values of the corrosion rate of inhibited system was found to be lower compared to the uninhibited system due to the inhibitive effect of the inhibitor. Increase in inhibitor concentration causes the increase in the rate at which the inhibitor molecules adsorbed on the surface of the aluminium thereby forming a barrier for charge and mass transfer which results into a decrease in the interaction between the metal surface and the corrosive media and also, reduces the rate of corrosion.

Table 1. Variation of Corrosion Parameters for Corrosion of Aluminium in $1.4 \mathrm{M} \mathrm{HCl}$ in the Absence and Presence of Different Concentrations of Inhibitor.

\begin{tabular}{|c|c|c|c|c|c|c|c|c|c|}
\hline \multirow{2}{*}{$\begin{array}{l}\text { Inhibitor } \\
\text { Conc. (M) }\end{array}$} & \multicolumn{3}{|c|}{ Corrosion rate $\left(\mathrm{mgcm}^{-2} \mathrm{~h}^{-1}\right)$} & \multicolumn{3}{|c|}{ Surface Coverage $(\theta)$} & \multicolumn{3}{|c|}{ Inhibition efficiency (\%) } \\
\hline & $308 \mathrm{~K}$ & $313 \mathrm{~K}$ & $318 \mathrm{~K}$ & $308 \mathrm{~K}$ & $313 \mathrm{~K}$ & $318 \mathrm{~K}$ & $308 \mathrm{~K}$ & $313 \mathrm{~K}$ & $318 \mathrm{~K}$ \\
\hline Blank & 19.527 & 21.290 & 25.138 & - & - & - & - & - & - \\
\hline 0.02 & 13.366 & 16.566 & 19.761 & 0.3155 & 0.2451 & 0.2131 & 31.55 & 24.51 & 21.31 \\
\hline 0.04 & 12.450 & 15.483 & 18.577 & 0.3624 & 0.2945 & 0.2602 & 36.24 & 29.45 & 26.02 \\
\hline 0.06 & 11.888 & 14.633 & 17.916 & 0.3911 & 0.3332 & 0.2866 & 39.11 & 33.32 & 28.66 \\
\hline 0.10 & 10.733 & 12.527 & 16.405 & 0.4503 & 0.4292 & 0.3468 & 45.03 & 42.92 & 34.68 \\
\hline
\end{tabular}

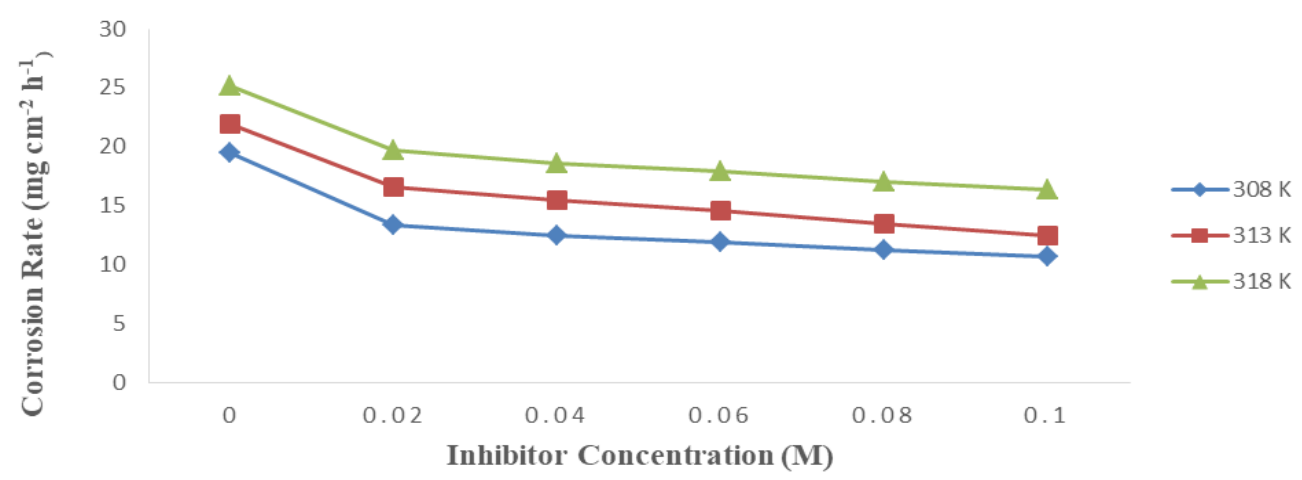

Fig.1. Variation of Corrosion Rate with Inhibitor Concentration for Al Corrosion in $1.4 \mathrm{M} \mathrm{HCl}$

Fig.1 shows the variation of inhibition efficiency against the different concentration of the inhibitor at 308,313 and $318 \mathrm{~K}$. The figure reveal that the inhibition efficiency increases with increase in the concentration of the inhibitor. This is due to the formation of more protective barrier film of the inhibitor molecules on the metal surface, which further supporting the inhibitive action of the inhibitor against the corrosion of the metal. Similar result was reported by Karthikaiselvi and Subhashini [15].

\subsection{Effect of Temperature on Corrosion Rate and Inhibition Efficiency}

The corrosion rates of aluminium in the absence and presence of various concentrations of the inhibitor at different temperatures was shown in Fig.3. The result obtained showed that the rate of corrosion of aluminium 
increased with increase in temperature. This phenomenon is due to the fact that chemical reaction rates normally increases when the temperature increase. Increase in temperature leads to increase in the average kinetic energy possessed by the reacting molecules thereby making the molecules to overcome the energy barrier and react faster [16].

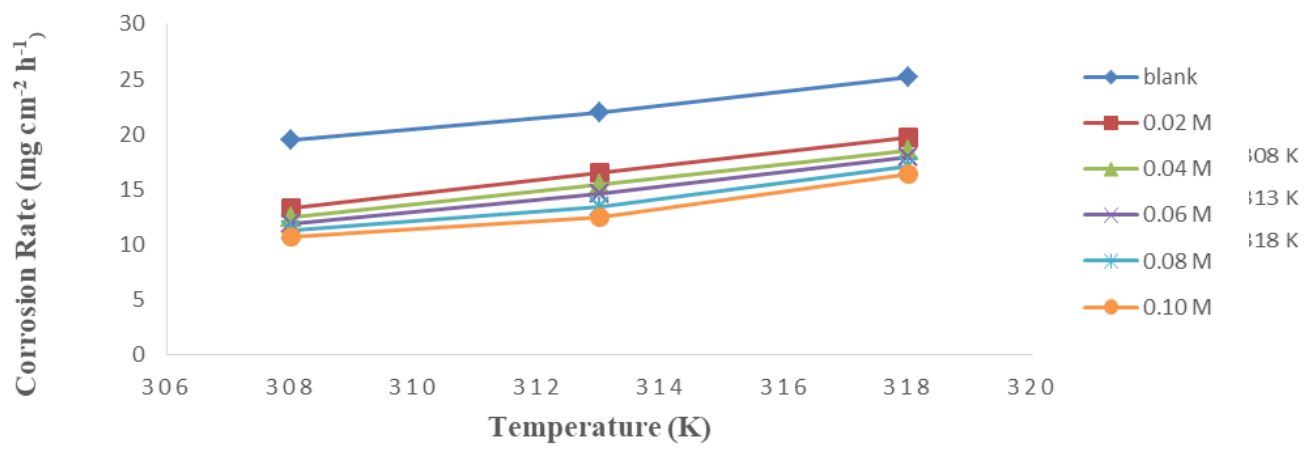

Fig.2. Variation of Inhibition Efficiency with Inhibitor Concentration for Al Corrosion in $1.4 \mathrm{M} \mathrm{HCl}$

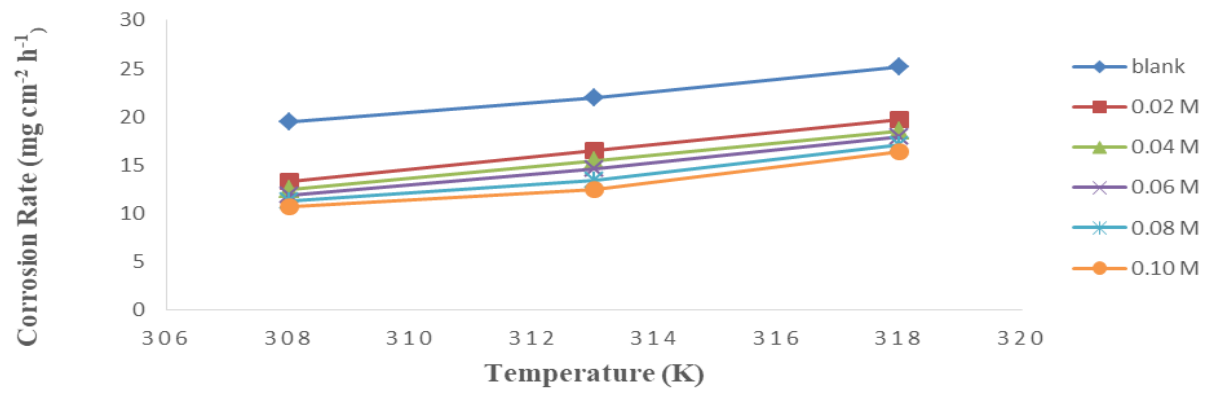

Fig.3. Variation of Corrosion Rate with Temperature for Al Corrosion in $1.4 \mathrm{M} \mathrm{HCl}$

Variation of inhibition efficiency with temperature at different concentration of the inhibitors is shown in Figure 4. The result obtained showed that the inhibition efficiency decreases with increase in temperature, this is due to the desorption of adsorbed inhibitor molecules from aluminium surface. The significant difference between values of inhibition efficiency of the given inhibitor obtained at the studied temperature suggested that the mechanism of adsorption of inhibitor on aluminum surface is consistent with physical adsorption mechanism. For a physical adsorption mechanism, inhibition efficiency of the inhibitor increases with decrease in temperature which mean physical adsorption mechanism occurred on the aluminium surface. Similar result was reported by reported by Eddy et al [17] and Abdallah [18].

\subsection{Adsorption behaviour}

To understand the mechanism of corrosion inhibition process, the adsorption behaviour of the adsorbate on the aluminium surface must be known. The information on the interaction between the metal surface and 
the inhibitor molecules can be provided by adsorption isotherm. The degree of surface coverage $(\Theta)$ for different concentrations of inhibitor was evaluated from weight loss measurements. The data was applied to various isotherms. Attempts were made to fit the experimental data into different isotherms. The result indicates that Langmuir adsorption isotherm model best described the adsorption characteristics of the inhibitor on the aluminium surface. Langmuir adsorption isotherm is the ideal adsorption isotherm for physical and chemical adsorption on a smooth surface, and it is valid for monolayer adsorption onto a surface containing a finite number of identical sites. According to this isotherm, the surface coverage $(\Theta)$ is related to the inhibitor concentration Cinh by the equation given below;

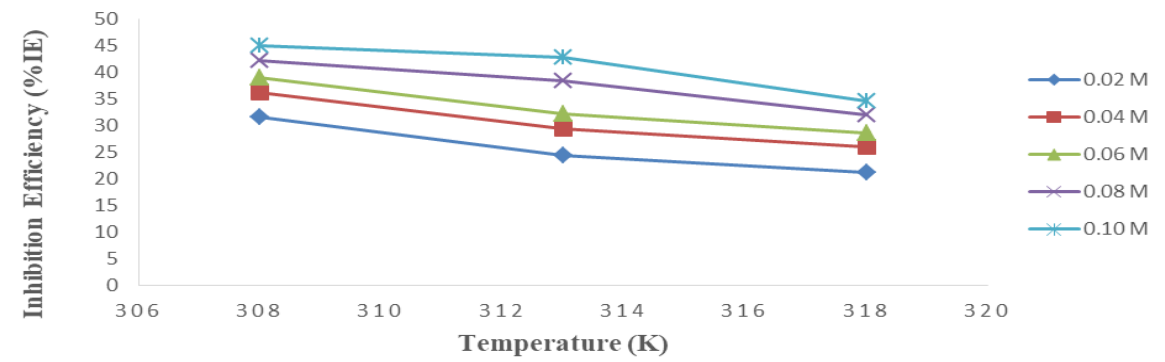

Fig.4. Variation of Inhibition Efficiency with Temperature for Al Corrosion in $1.4 \mathrm{M} \mathrm{HCl}$

$$
\frac{\mathrm{C}_{i n h}}{\theta}=\frac{1}{K_{a d s}}+\mathrm{C}_{\mathrm{inh}}
$$

Where $\mathrm{K}_{\mathrm{ads}}$ is the adsorption equilibrium constant, $\mathrm{C}_{\mathrm{inh}}$ is the inhibitor concentration in the solution, and $\Theta$ is the surface coverage. The plot of $\mathrm{C}_{\mathrm{inh}} / \Theta$ against $\mathrm{C}_{\mathrm{inh}}$ gave a straight line with slope equal to unity and $\mathrm{R}^{2}$ close to 1 indicating that the adsorption of the inhibitor on the surface of aluminium is consistent with Langmuir adsorption isotherm. The correlation coefficient $\left(\mathrm{R}^{2}\right)$ and adsorption equilibrium constant $\left(\mathrm{K}_{\mathrm{ads}}\right)$ are presented in Table 3.

\subsection{Kinetic Study}

\subsubsection{Rate Constant $(\mathrm{k})$ and Half Life $\left(\mathrm{t}_{1 / 2}\right)$}

Kinetic analysis of the data is considered necessary since corrosion reaction is a heterogeneous process that composed of anodic and cathodic reactions with the same or different rate. In this current study, the initial weight of aluminum specimen at given time, $t$ is designated as $\mathrm{W}_{\mathrm{i}}$, the weight loss is $\mathrm{W}_{\mathrm{L}}$ and the weight change at time $t$ is $\left(\mathrm{W}_{\mathrm{i}}-\mathrm{W}_{\mathrm{L}}\right)$, while $\mathrm{k}_{1}$ is the first order rate constant.

$$
\ln \left(\mathrm{Wi}-\mathrm{W}_{\mathrm{L}}\right)=-\mathrm{k}_{1} \mathrm{t}+\ln \mathrm{W}_{\mathrm{L}}
$$

The plots of $\ln \left(\mathrm{Wi}-\mathrm{W}_{\mathrm{L}}\right)$ against time $(\mathrm{hr})$ at $308 \mathrm{~K}$ showed a linear variation which confirms a first order reaction kinetics with respect to the corrosion of aluminium in $1.4 \mathrm{M} \mathrm{HCl}$ solutions in the presence of the inhibitor. The first order reaction rate constants $\left(\mathrm{k}_{1}\right)$ calculated from the slope of the plot are presented in Table 2. The result shows that the values of rate constant $\left(\mathrm{k}_{1}\right)$ for the corrosion of aluminium was found to be higher in the case of uninhibited acid solution than inhibited acid solution. This confirmed the inhibition of 
aluminium corrosion in acids solution by the presence of inhibitors.

The half-life $\left(t_{1 / 2}\right)$ was calculated from equation 7

$$
\mathrm{t}_{1 / 2}=0.693 / \mathrm{k}
$$

From Table 2, the values of the half-lives $\left(t_{1 / 2}\right)$ increased from uninhibited solution to inhibited solution. The increase in half-lives $\left(\mathrm{t}_{1 / 2}\right)$ in the presence of the inhibitor compared to the uninhibited solution support the earlier results that corrosion rate decreases in the presence of the inhibitor compared to the uninhibited solution. It can also be seen that as the concentration of the inhibitor increases, the half-life also increases which results into a decrease in the corrosion rate suggesting that more protection of the aluminium by the inhibitor has been established.

3.4.2 Activation Energy (Ea)

The rate of most chemical reactions increases with temperature following Arrhenius equation [17]. In the case of the electrochemical reactions, temperature favors the kinetics of corrosion reactions and more specifically, the anodic dissolution of the metal. The activation energy of the corrosion process was calculated using the Arrhenius law equation

$$
\ln (\mathrm{C} . \mathrm{R})=\ln \mathrm{B}-\frac{\mathrm{E}_{a}}{R T}
$$

Where $\mathrm{B}$ is a constant which depends on the metal type, $\mathrm{R}$ is the universal gas constant, and $\mathrm{T}$ is the absolute temperature. The plot of $\ln$ (C.R) Vs reciprocal of absolute temperature (1/T) gave a straight line with slope $=-\mathrm{Ea} / \mathrm{R}$, from which the activation energy values for the corrosion process was calculated. The calculated activation energy values were tabulated in Table 2 . The activation energy value in uninhibited hydrochloric acid solution is low com $\neg$ pared to inhibited hydrochloric acid solution. The higher activa $\neg$ tion energy values in the presence of inhibitor molecules strongly support the results of corrosion rate studies and confirms the physisorption mechanism (inhibition efficiency decreases with an increase in solution temperature).

Table 2. Kinetic Parameters for Aluminium Corrosion with and without various inhibitor concentrations

\begin{tabular}{cccc}
\hline $\begin{array}{c}\text { Inhibitor Concentration } \\
(\mathbf{M})\end{array}$ & $\begin{array}{c}\text { Activation Energy } \\
\left(\mathbf{k j ~ m o l}^{-\mathbf{1}}\right)\end{array}$ & Rate Const. $\left(\mathbf{k} \times \mathbf{1 0}^{-\mathbf{3}}\right)\left(\mathbf{h o u r}^{\mathbf{- 1}}\right)$ & Half-life (hours) \\
\hline Blank & 20.55 & 144.39 & 4.79 \\
0.02 & 31.84 & 91.75 & 7.55 \\
0.04 & 32.60 & 84.59 & 8.19 \\
0.06 & 33.39 & 80.28 & 9.63 \\
0.08 & 33.81 & 75.56 & 9.17 \\
0.10 & 34.49 & 71.57 & 9.68 \\
\hline
\end{tabular}

\subsection{Thermodynamic Study}

The Thermodynamic parameters like enthalpy of activation $(\Delta \mathrm{Ha})$ and entropy of activation $(\Delta \mathrm{Sa})$ were calculated using the transition state equation. The linear form of transition state equation is given by the equation below 


$$
\ln \left(\frac{\mathrm{C}_{\mathrm{R}}}{\mathrm{T}}\right)=\ln \left(\frac{\mathrm{R}}{\mathrm{Nh}}\right)+\left(\frac{\Delta \mathrm{S}_{\mathrm{a}}}{\mathrm{R}}\right)-\left(\frac{\Delta \mathrm{H}_{\mathrm{a}}}{\mathrm{RT}}\right)
$$

Where h is Plank's constant and N is Avagadro's number. A plot of $\ln (\mathrm{C} . \mathrm{R} / \mathrm{T})$ vs $1 / \mathrm{T}$ gave a straight line with slope $=-\Delta \mathrm{Ha} / \mathrm{T}$ and intercept $=\ln (\mathrm{R} / \mathrm{Nh})+\Delta \mathrm{Sa} / \mathrm{R}$. The calculated values of enthalpy of activation $(\Delta \mathrm{Ha})$ and entropy of activation $(\Delta \mathrm{Sa})$ are tabulated in Table 3 . The positive signs of the enthalpies reflect the endothermic nature of the aluminium dissolution process. Large negative values of entropies show that the activated complex in the rate determining step is an association rather than dissociation step meaning that a decrease in disordering takes place on going from reactants to the activated complex.

Table 3. Enthalpy and Entropy change of the reaction process with various concentrations of the inhibitor

\begin{tabular}{|c|c|c|c|}
\hline $\begin{array}{l}\text { Inhibitor } \\
\text { (M) }\end{array}$ & Concentration & $\Delta \mathrm{H}\left(\mathrm{kJ} \mathrm{mol}^{-1}\right)$ & $-\Delta \mathrm{S}\left(\mathrm{kJ} \mathrm{mol}^{-1} \mathrm{k}^{-1}\right)$ \\
\hline & blank & 17.95 & 219.63 \\
\hline & 0.02 & 29.24 & 186.05 \\
\hline & 0.04 & 30.00 & 184.19 \\
\hline & 0.06 & 30.79 & 182.04 \\
\hline & 0.08 & 31.21 & 181.21 \\
\hline & 0.10 & 31.89 & 179.48 \\
\hline
\end{tabular}

\subsubsection{Free Energy of Adsorption ( $\Delta$ Gads,}

The free energy of adsorption ( $\Delta$ Gads) is related to adsorption equilibrium constant (Kads) by the equation given below

$$
\Delta \mathrm{G}_{\mathrm{ads}}=-\mathrm{RT} \ln \left(55.5 \mathrm{~K}_{\mathrm{ads}}\right)
$$

The negative values of free energy of adsorption (Gads) presented in Table 4 suggests that the inhibitor molecules are strongly adsorbed on the metal surface, the values also shows a spontaneous adsorption of the inhibitor molecules mainly characterized by the strong interactions with the metal surface. The values of $\Delta$ Gads around $-20 \mathrm{kJmol}-1$ are consistent with electrostatic interaction between charged molecules and a charged metal indicating physical adsorption (physisorption) mechanism, while those around $-40 \mathrm{kJmol}-1$ involve charge sharing or transfer of electrons to form a co-ordinate type of bond otherwise known as chemical adsorption (chemisorption) mechanism. In the present study, the values of $\Delta$ Gads are less than -20 kJ mol-1 which indicate that the adsorption of the inhibitor on the aluminium surface confirms a physical adsorption mechanism. Similar result was reported by Vimala et al [19].

Table 4. Adsorption Parameters Deduced from Langmuir Adsorption Isotherm for Corrosion Inhibition of Aluminium.

\begin{tabular}{cccc}
\hline Temperature (K) & $\mathbf{R}^{\mathbf{2}}$ & $\mathbf{K}_{\text {ads }}$ & $\Delta \mathbf{G}\left(\mathbf{k J} \mathbf{~ m o l}^{-\mathbf{1}}\right)$ \\
\hline 308 & 0.9943 & 19.90 & -17.94 \\
313 & 0.9918 & 18.69 & -18.07 \\
318 & 0.9986 & 18.08 & -18.27 \\
\hline
\end{tabular}

3.6 Infrared Spectroscopy Analysis Results 
The FTIR spectra of the inhibitor (benzaldehyde) and that of the corrosion product are presented in Figures 5 and 6 . The analysis of the inhibitor presented in Figure 5 revealed the presence of carbonyl group $\mathrm{C}=\mathrm{O}$ stretch, aromatic $\mathrm{C}-\mathrm{H}$ stretch and aldehyde $\mathrm{C}-\mathrm{H}$ stretch. The analysis of the corrosion product presented in Figure 6 shows the appearance of carbonyl group $\mathrm{C}=\mathrm{O}$ stretch, aromatic $\mathrm{C}-\mathrm{H}$ stretch and aldehyde $\mathrm{C}-\mathrm{H}$ stretch and this confirmed that the adsorption of the inhibitor on the metal surface took place.

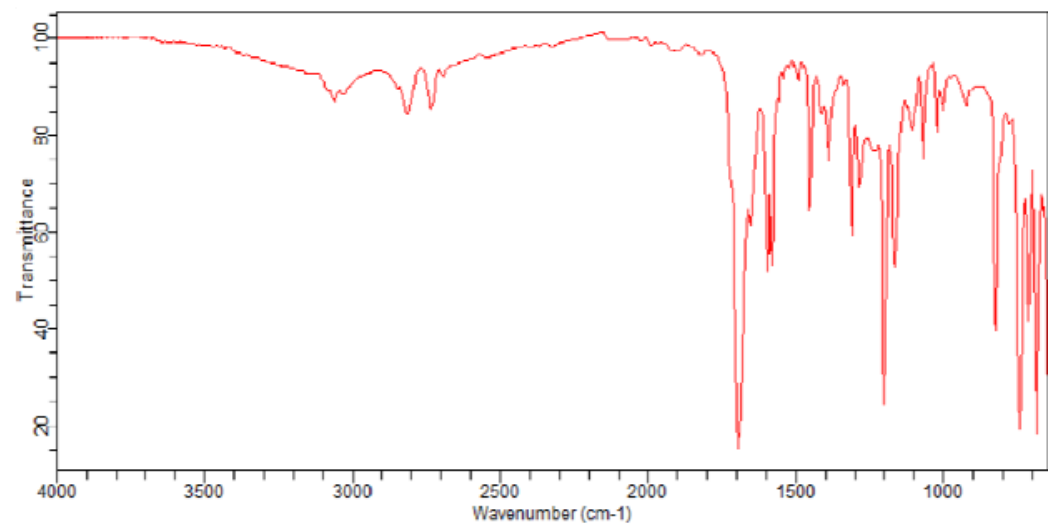

Fig.5. FT-IR Spectra of Benzaldehyde

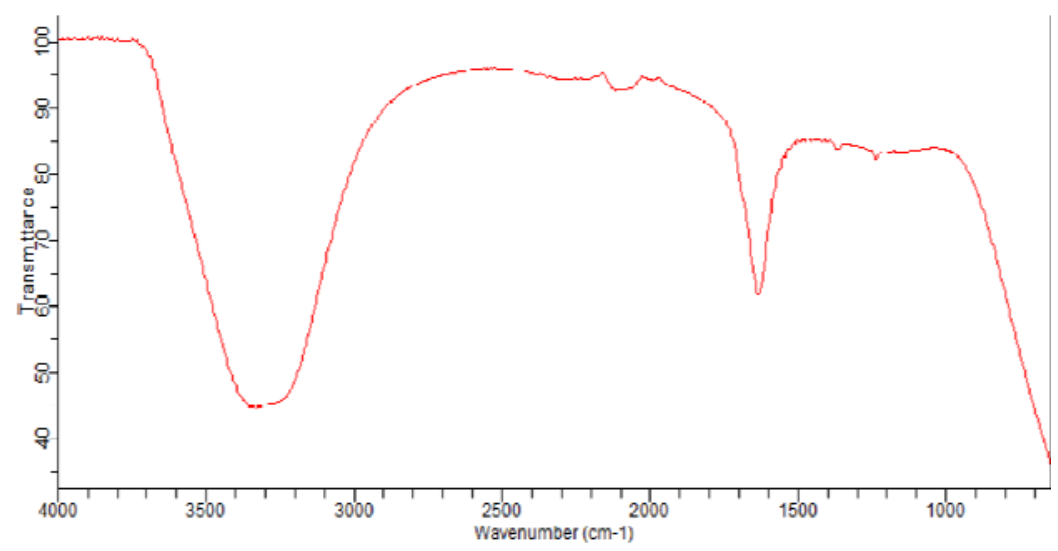

Fig.6. FT-IR Spectra of Aluminium in $1.4 \mathrm{M} \mathrm{HCl}$ with $0.1 \mathrm{M}$ Benzaldehyde

\section{Conclusion}

From the result of this study it can be concluded that benzaldehyde served as good inhibitor in reducing the rate of hydrochloric acid attack on the surface of aluminium, by electrostatic interaction between the charged inhibitor molecules and the charged surface of aluminium. This make the adsorption process occurred through physical adsorption mechanism (physisorption). The calculated values of Gibbs free energy of adsorption, enthalpy and activation energy also support the physisorption mechanism of the process. The presence of 
functional group of the inhibitor molecule in the FT-IR of the corrosion product confirmed that the adsorption of the inhibitor on the metal surface took place. The adsorption of different concentrations of the inhibitor on the surface of the aluminium in hydrochloric acid followed Langmuir adsorption isotherm.

This work is only limited to the thermodyamic and kietic study on the corrosion of aluminium in hydrochloric acid using benzaldehyde as corrosion inhibitor using only weight loss method. It's recommended that the feature work on the corrosion inhibition effect of benzaldehyde to include other methods of corrosion inhibition analysis such as gravimetric, thermometric, electrochemical impedance spectroscopy e.t.c.

\section{References}

[1] Abdel-Gaber, A. M., Abd-El-Nabey, B. A., Sidahmed, I. M. El-Zayady, A. M. and Saadawy, M. (2006). Kinetics and Thermodynamics of Aluminium Dissolution in 1.0 M Sulphuric Acid Containing Chloride Ions. Mater., Chem. Phys., 98: 291-297.

[2] Stansbury, E. E. and Buchanan, R. A. (2000). Fundamentals of Electrochemical Corrosion, ASM International Materials Park, USA.

[3] Li, X., Deng, S. and Fu, H. (2011). Inhibition by Tetradecylpyridinium Bromide of the Corrosion of Aluminium, Inhibition by Tetradecylpyridinium Bromide of the Corrosion of Aluminium in Hydrochloric Acid Solution. Corros. Sci., 53:1529-1536.

[4] Musa, A. Y. Kadhum, A. A. H., Mohamad, A. B., Takriff, M. S. and Chee, E. P. (2012) Inhibition of Aluminium Corrosion by Phthalazinone Synergistic Effect of Halide Ion in 1.0 M HCl. Curr. Appl. Phys., 12, 325-330.

[5] Lopez-Sesenes R, Gonzalez-Rodriguez, J. G., Casales, M., Martinez, L., and Sanchez-Ghenno, J. C. (2011). Corrosion Inhibition of Carbon Steel in 0.5 M HCl by Monopropianate. Int. J. Electrochem. Sci., 6: 1772-1784. [6] Awad, H. S. and Gawad, S. A. (2005). Mechanism of inhibition of iron corrosion in hydrochloric acid by pyrimidine and series of its derivatives. Anti-Corros. Method Mater.,52: 328.

[7] Chetouani, A., Aouniti, A., Hammouti, B., Benchat, N., Benhadda, T. and Kertit, S. (2003). Corrosion inhibitors for iron in hydrochloride acid solution by newly synthesized pyridazine derivatives. Corros. Sci., 45: 1675-1684.

[8] Sherif, E.S.M. (2012). Electrochemical and Gravimetric Study on the Corrosion and Corrosion Inhibition of Pure Copper in Sodium Chloride Solutions by Two Azole Derivatives. International Journal of Electrochemical Science. 7: 1482-1859.

[9] Fouda, A. S., Gadow, H. S. and Shalabi, K. (2015). Chemical and Electrochemical Investigations of Coffee Husk as Green Corrosion Inhibitor for Aluminum in Hydrochloric Acid Solutions. . International Journal of Innovative Research in Science. 23(1) 28-45.

[10] Karthikeyan, S., Lakshmi, N.V. and Arivazhagan, N. (2013). The Corrosion Inhibition of Aluminium in $3.5 \% \mathrm{NaCl}$ by Diisopropyl Thiourea. International Journal of Chemical Technology and Research. 5(4): 1959-1963.

[11] Anand, B. (2013). Effect Formazan of Benzaldehyde as Corrosion Inhibitor on Preventing the Mild Steel Corrosion in Acidic Medium. Chemical Science Transactions. 2(4), 1126-1135.

[12] Arvnabh, M., Godhani, D.R. And Sanghani, A. (2011). Diamines as corrosion inhibitors for aluminium alloy in organic acid. The Asian Journal of Experimental Chemistry. 6(1): 38-41.

[13] Yadav, M., Kumar, S., Sharma, U. and Yadav, P.N. (2013). Substituted amines as corrosion inhibitors for 
N80 steel in 15\% HCl. Journal of Material and Environmental Science. 4(5): 691-700.

[14] Husaini, M., Usman, B. and Ibrahim, M. B. (2019). Study of corrosion inhibition of aluminium in nitric acid solution using Anisaldehyde (4 - methoxy benzaldehyde) as inhibitor. Algerian Journal of Engineering and Technology.

[15] Karthikaiselvi, R. and Subhashini, S. (2013). Study of adsorption properties and inhibition of mild steel corrosion in hydrochloric acid media by water soluble composite poly (vinyl alcohol-o-methoxy aniline). J. Assoc. Arab Univ. Basic Appl. Sci., http://dx.doi.org/ 10.1016/j.jaubas.

[16] Musa, H., Bishir, U. and Muhammad. B. I. (2018). Evaluation of corrosion behaviour of aluminium in different environment. Bayero Journal of Pure and Applied Sciences. 11(1): 88-92.

[17] Eddy, N.O., Ibok, U.J., Ebenso, E.E., El Nemr, A. and El Ashry, S.H. (2009). Quantum chemical study of the inhibition of the corrosion of mild steel in H2SO4 by some antibiotics. J. Mol. Model. 15(9): 1085- 1092.

[18] Abdallah, M. (2004). Antibacterial drugs as corrosion inhibitors for corrosion of aluminium in $\mathrm{HCl}$ solution. Corros. Sci. 46: 1981-1996.

[19] Vimala, J. R., Rose, A. L. and Raja, S. (2011). Cassia auriculata extract as corrosion inhibitor for mild steel in acid medium. Int. J. Chem.Tech. Res., 3(4): 1791-1801.

\section{Author's Profile}

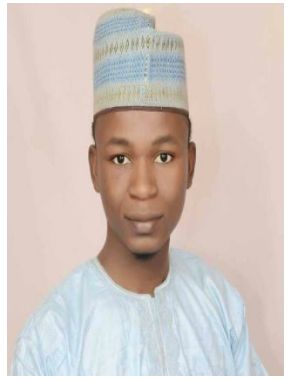

Musa Husaini Studies: B.Sc Chemistry (Umaru Musa Yaradua University Katsina, Nigeria); M.Sc Physical Chemistry (Bayero University Kano, Nigeria). Currently working on corrosion protection of metallic substances.

How to cite this paper: Musa Husaini. "Thermodyamic and Kietic Study on the Corrosion of Aluminium in Hydrochloric Acid using Benzaldehyde as Corrosion Inhibitor", International Journal of Engineering and Manufacturing(IJEM), Vol.9, No.6, pp.53-64, 2019. DOI: 10.5815/ijem.2019.06.05. 